5. FINA. Open water rulebook. 2013. Available at: http:// www.fina.org. Accessed December 4, 2013.

\section{Regarding the Wilderness Medical Society Practice Guidelines for Heat-Related Illness}

\section{To the Editor:}

Exercise-associated hyponatremia $(\mathrm{EAH})$ is recognized as a potential and serious complication from improper hydration strategies during endurance exercise. Several deaths attributable to EAH have been reported, ${ }^{1}$ with morbidity and mortality likely exacerbated by inappropriate medical management with isotonic or hypotonic fluids under the clinical presumption that the athlete was dehydrated. ${ }^{1,2}$ It is now clear that isotonic and hypotonic fluids will worsen cerebral encephalopathy in cases of dilutional EAH and, at a minimum, result in a prolonged recovery. ${ }^{2}$ Proper management of symptomatic EAH from either a dilutional or depletional mechanism is with hypertonic saline as discussed in the recently published Wilderness Medical Society (WMS) Practice Guidelines for EAH. ${ }^{3}$ Furthermore, the risks and benefits of intravenous isotonic fluid administration in symptomatic EAH have been outlined in a response to a letter to the editor. ${ }^{4}$ The physiological basis for avoiding isotonic fluids in EAH becomes evident when it is recognized that the usual pathophysiology of nonosmotic arginine vasopressin secretion in both dilutional and depletional EAH will facilitate urinary water retention and urinary sodium excretion, ${ }^{1,2}$ the latter possibly enhanced by circulating brain natriuretic hormone. ${ }^{5}$ Thus, in these cases of EAH as a variant of the syndrome of inappropriate secretion of antidiuretic hormone (SIADH), isotonic fluid administration will either worsen the condition or prolong recovery as originally described in $1967 .^{1}$

We are concerned that the recently published WMS Practice Guidelines for heat-related illness ${ }^{6}$ do not address the potential consequences from the provision of isotonic fluids in the presence of dilutional EAH from an SIADH-like mechanism. As discussed in the WMS Practice Guidelines for EAH and acknowledged in the Practice Guidelines for heat-related illness, the constellation of symptoms of EAH overlap with those of dehydration and heat illness. As such, appropriate diagnosis in the field by clinical symptoms alone is rarely possible, and could result in inappropriate treatment leading to potentially disastrous clinical outcomes. $^{1,2}$ Therefore, we advocate caution when using intravenous isotonic or hypotonic fluids in the field when blood sodium concentration is unknown because the benefits may be negligible ${ }^{7}$ and the risks of either worsening or delaying recovery from EAH will be significantly increased. ${ }^{1,2}$ Furthermore, when treating endurance athletes in the field with intravenous isotonic or hypotonic fluids for presumed dehydration, it is prudent to have immediate access to hypertonic saline should the patient deteriorate.

We must also express concern with the WMS Practice Guidelines for heat-related illness relative to the recommendations that promote overhydration. Without providing references, the document indicates that "heat" cramps are related to hydration or electrolyte disturbances and that treatment includes oral isotonic or hypertonic salt or electrolyte replacement. First, to label exercise-associated muscle cramps (EAMC) as heat cramps is incorrect. EAMC can occur in any environmental conditions, not only during exercise in hot, humid environmental conditions. Second, there is very strong evidence from a number of prospective cohort studies and a recent laboratory study that convincingly show that neither fluid and electrolyte disturbances nor dehydration are associated with EAMC. ${ }^{8}$ Rather, this work indicates that fatiguing exercise, past history of EAMC, underlying muscle or tendon injury, and possibly genetic predisposition are more predictive of the risk of EAMC. ${ }^{8}$ Furthermore, these data can best be explained by a common hypothesis whereby a final common pathophysiological pathway of altered neuromuscular control is responsible for the development of EAMC in athletes. ${ }^{8}$ As such, the recommendation to treat muscle cramps with oral hydration, salt, or electrolyte replacement is not only unfounded, but may encourage overhydration with the possibility of inducing dilutional EAH.

We also take issue with the general recommendation to avoid loss of over $2 \%$ of body weight during exercise. To understand why, consider the basic physiological principle that water is bound to glycogen, and that this water is released as glycogen is oxidized. It has been estimated that approximately $1.5 \mathrm{~kg}$ of water is bound to glycogen, and when considering the weight associated with substrate utilization, a weight loss of over $2 \mathrm{~kg}$ (approximately 3\%) is required to maintain euhydration during prolonged exercise in a $70-\mathrm{kg}$ athlete. ${ }^{9}$ It is also evident that endurance athletes are well tolerant of weight losses of at least $8 \% .^{9,10}$ Furthermore, body weight losses greater than $3 \%$ to $4 \%$ have been shown to be common among athletes with the best performances in various endurance events. ${ }^{10}$ Therefore, a general recommendation to avoid more than $2 \%$ weight loss during prolonged exercise in a competitive field setting is unsupported and could precipitate the development of dilutional EAH.

We raise legitimate concerns about overhydration because symptomatic EAH is generally associated with 
water retention from the overconsumption of fluids or sustained consumption beyond the capacity for urinary free water excretion. EAH is not uncommon with considerable weight loss in some environments, ${ }^{10}$ but symptomatic cases are generally associated with weight gain and weight loss of less than $3 \%$ of body weight. ${ }^{9}$ As noted in the WMS Practice Guidelines for EAH, athletes should be encouraged to drink to thirst, and strive for a weight loss of $2 \%$ to $4 \%$ during prolonged exercise. ${ }^{3}$ Recommendations to avoid more than $2 \%$ weight loss serve to promote the overconsumption of fluids.

The issues we raise are not trivial, as the failure to prevent and appropriately manage EAH can mean the difference between life and death. We hope that by highlighting the potential dangers of overhydration and use of intravenous isotonic or hypotonic saline in those with EAH, your readers will be better prepared to appropriately advise endurance athletes about hydration and make better medical decisions when faced with a potential case of EAH.

\author{
Martin D. Hoffman, MD \\ Sacramento, $C A$ \\ Tamara Hew-Butler, DPM, PhD \\ Rochester, MI \\ Martin Schwellnus, MD, PhD, MSc \\ Cape Town, South Africa
}

\section{References}

1. Siegel AJ, Verbalis JG, Clement S, et al. Hyponatremia in marathon runners due to inappropriate arginine vasopressin secretion. Am J Med. 2007;120(461):e11-e17.

2. Hew-Butler TD, Boulter J, Bhorat R, Noakes TD. Avoid adding insult to injury-correct management of sick female endurance athletes. S Afr Med J. 2012;102:927-930.

3. Bennett BL, Hew-Butler T, Hoffman MD, Rogers IR, Rosner MH. Wilderness Medical Society Practice Guidelines for treatment of exercise-associated hyponatremia. Wilderness Environ Med. 2013;24:228-240.

4. Bennett BL, Hew-Butler T, Hoffman MD, Rogers IR, Rosner MH. In reply to Clinical Practice Guidelines for treatment of exercise-associated hyponatremia. Wilderness Environ Med. 2013;24:468-471.

5. Harris G, Reid S, Sikaris K, McCrory P. Hyponatremia is associated with higher NT-proBNP than normonatremia after prolonged exercise. Clin J Sport Med. 2012;22:488-494.

6. Lipman GS, Eifling KP, Ellis MA, Gaudio FG, Otten EM, Grissom CK. Wilderness Medical Society Practice Guidelines for the prevention and treatment of heat-related illness. Wilderness Environ Med. 2013;24:351-361.
7. Noakes TD. Hyponatremia in distance athletes: pulling the IV on the 'dehydration myth.' Phys Sportsmed. 2000;28:71-76.

8. Schwellnus MP. Cause of exercise associated muscle cramps (EAMC) - altered neuromuscular control, dehydration or electrolyte depletion? Br J Sports Med. 2009; 43:401-408.

9. Noakes TD, Sharwood K, Speedy D, et al. Three independent biological mechanisms cause exerciseassociated hyponatremia: evidence from 2,135 weighed competitive athletic performances. Proc Natl Acad Sci U S A. 2005; 102:18550-18555.

10. Hoffman MD, Hew-Butler T, Stuempfle KJ. Exerciseassociated hyponatremia and hydration status in 161-km ultramarathoners. Med Sci Sports Exerc. 2013;45:784-791.

The Importance of Keeping Cool: Reply Regarding the Wilderness Medical Society Practice Guidelines for the Prevention and Treatment of HeatRelated Illness

To the Editor:

As there is significant morbidity and mortality from heat stroke in wilderness activities, mass gatherings, and regional heat waves, our recently published Wilderness Medical Society's Practice Guidelines for the Prevention and Treatment of Heat-Related Illness ${ }^{1}$ were intended to elucidate and provide guidance in the appropriate recognition and management of the spectrum of disease of heat injury. We emphasized the need for rapid recognition and implementation of cooling treatments, highlighted the safety and evidence-based recommendation for treatment by cold-water immersion, and stressed the importance of continuation of cooling treatments until the victim's core temperature could be stabilized, as injury is directly related to both magnitude and duration of hyperthermia. The concern of Hoffman et al that rapid cooling and volume replacement in an endurance athlete suffering from heat stroke should be withheld owing to the possible coexistence of exerciseassociated hyponatremia (EAH) is a somewhat myopic perspective that could inadvertently lead to dangerous mismanagement of heat-related illnesses.

The majority of athletes with exercise-induced heat illness are dehydrated with minimal dysnatremia. ${ }^{2}$ When hyperpyrexia occurs or an altered sensorium becomes evident, the severe disease states manifest and become distinctive. The apparent confusion by Hoffman et al is likely based on their erroneous presumption that heat stroke and severe EAH (EAH with encephalopathy) often overlap, because there is a lack of any evidence supporting the coexistence of these 2 severe diseases. Heat stroke is marked by multiorgan dysfunction, 